ORIGINAL PROF-2288

\title{
Clinical Manifestations of Goldenhar Syndrome
}

\author{
Dr. Fuad Ahmad Khan Niazi, Dr. Abdul Munim Khan, Dr. Manal Niazi, Prof. Muhammad Afzal Khan Niazi
}

ABSTRACT..... Goldenhar syndrome is a congenital disorder with diverse clinical presentation. This case series describes various clinical manifestations of Goldenhar Syndrome. Materials and Methods: 11 patients at least two of the described features of Goldenhar syndrome were randomly included in the study to form a case series. Clinical data of all these subjects was documented, described in percentages and analyzed. Results: Out of the 11 patients studied, 6 were females and 5 males. Mean age was 7.3 years (ranging from 1 year to 23 years). 7 (63.6\%) patients had a limbal dermoid, 6 (54.5\%) patient had mandibular hypoplasia, whereas only 3(27.3\%) presented with hemifacial microsomia. Spinal defects and polydactyly were present in (36.4\%) patients each. There was one patient (9\%) who had both microphthalmia and lid coloboma in addition to limbal dermoid. Similarly, renal defect (atrophic kidney), cleft upper lip and palate and CNS defect (7th nerve palsy) were present in one patient (9\%) each. None of our patients had either cardiac or GIT defect. Conclusions: In our study we were able to document the variable manifestations of Goldenhar syndrome with their frequencies. This knowledge base will help us in planning treatment and assistance strategies or these patients.

Key words: Goldenhar, Syndrome, Limbal, Dermoid

Article Citation

Niazi FAK, Khan AM, Niazi M, Niazi MAK. Clinical Manifestation of Goldenhar Syndrome. Professional Med J. 2013;20(4) 617-622.

\section{INTRODUCTION}

Goldenhar syndrome, first described by Dr Maurice Goldenhar in 1952, is an uncommon (1 in 3500 to 1 in 7000 live births) congenital disorder also frequently referred to as oculo-auriculo-vertebral disease or spectrum ${ }^{1,2}$. It is characterized by variable clinical presentations which may include limbal dermoids, microtia, preauricular skin tags, deafness, mandibular hypoplasia, hemifacial microsomia, spinal, cardiac, renal and gastrointestinal defects, and very rarely, polydactyly ${ }^{3,4,5}$. Most cases are unilateral, but bilateral cases have been reported. The anomalies of this syndrome are probably caused by development defects of the first and second brachial arches during the first gestational trimester ${ }^{6}$. Although, mostly it occurs sporadically, in rare instances an autosomal inheritance pattern has been observed ${ }^{3}$. A particular patient may have any one or more of the above defects and it is very common to miss certain hidden defects in these patients due to lack of detailed knowledge of the disease. The purpose of our study was to evaluate a series of patients with Goldenhar syndrome and determine the various modes of clinical presentation and associated defects in these patients, thereby enhancing the knowledge base, supporting and facilitating doctors, especially Ophthalmologists, in proper management of these patients including timely referral for multisystem care.

\section{MATERIALS \& METHODS}

Patients presenting with a limbal ocular dermoid, microtia, preauricular skin tag or hemifacial microsomia presenting in the eye department of Holy Family Hospital, Rawalpindi, Pakistan and Prof Afzal Niazi's Eye Surgery Hospital with same or other unrelated complaints were randomly included in the study from 2004 to 2012 after taking proper consent. 32 such patients were subjected to thorough clinical history and examination, X-ray Chest, X-ray Spine, Xray skull, ECG and abdominal ultrasound. 11 Patients with at least two of the described features of Goldenhar syndrome were included whereas rest of the patients were discarded from the study to form a case series. Clinical data of all these subjects was documented, described in percentages and analyzed. 


\section{RESULTS}

Out of the 11 patients studied, 6 were females and 5 males. Mean age was 7.3 years (ranging from 1 year to 23 years). 7 (63.6\%) patients had a limbal dermoid, $6(54.5 \%)$ had preauricular skin tags and $5(45.4 \%)$ had microtia. 6(54.5\%) patient had mandibular hypoplasia, whereas only $3(27.3 \%)$ presented with hemifacial microsomia. Spinal defects and polydactyly were present in $4(36.4 \%)$ patients each. There was one patient (9\%) who had both microphthalmia and lid coloboma in addition to limbal dermoid. Similarly, renal defect (atrophic kidney), cleft upper lip and palate and CNS defect (7th nerve palsy) were present in one patient $(9 \%)$ each. None of our patients had either cardiac or GIT defect.

\section{DISCUSSION}

Goldenhar syndrome or oculo-auriculo-vertebral spectrum is a congenital disorder with diverse clinical phonotypical presentation because of heterogeneous etiological factors. No established etiology has been identified so far and it is believed that various factors contribute in causing the condition, hence, the marked variability in presentation ${ }^{7}$. Hartsfield in 2007 suggested some form of vascular abnormality or disturbance of the neural crest during first few weeks of embryogenesis involving the first and second branchial arches as responsible for the condition. Although none of our patients had family history, some form of autosomal recessive inheritance has been reported. We also did not find any significant difference in gender distribution as previously described to be more frequent in males in a ratio of $3: 2^{2}$. Engiz et al[8] in their 31 patients also showed almost equal male female ratio.

In our series of patients $63.6 \%$ had a limbal dermoid and that was also their main reason for presenting to us. This was similar to the $65 \%$ in 6 patients reported by Martelli et al ${ }^{9}$ and and $66 \%$ in a case series of 17 Greek patients ${ }^{5}$. It was significantly different, however, from the $39 \%$ according to Engiz et al ${ }^{8}$.
Limbal dermoid is an uncommon congenital disorder (a choristoma) characterized by a round or oval swelling of variable size, pinkish white to yellowish brown in color, present anywhere on the limbus, more commonly inferotemporally and involving adjacent structures. Although present since birth it may increase in size and darken in color with age therefore explaining the sometimes late presentation in second or third decade of life. It may cause not only marked decrease in vision but also a cosmetic blemish resulting in significant panic among the parents. Surgical excision is the treatment of choice for such lesions. These lesions may occasionally be associated with other ocular anomalies like lid coloboma and microphthalmos ${ }^{9}$.

Second most common feature among our patients was preauricular skin tags and hemifacial microsomia (54.5\%). $45.4 \%$ had microtia. Microtia is the rudimentary development or underdevelopment of the ear resulting in deafness. Interestingly all the patients had atleast one ear abnormality. Tasse et al ${ }^{4}$ also described $100 \%$ ear abnormality in their patients. Martelli et al ${ }^{9}$ reported all, except one, had auricular pathology. In the 17 Greek patients studied by Touliatou et al, auricular defects formed the most common presentation $(94 \%)^{5}$. Engiz et al also confirmed the presence of preauricular skin tags in $90 \%$ and microtia in $52 \%$ of their 31 patients. Traditionally ear abnormalities are the most common presenting feature which generally prompts further investigations leading to discovery of associated pathologies. Many of these patients are frequently associated with deafness which is usually unilateral but can be bilateral occasionally ${ }^{10}$. This necessitates mandatory referral to an otologist for diagnosis and treatment of potential deafness and possible reconstruction procedures.

Interestingly, according to Touliatou et al, facial defects and asymmetry (76\%) form the second most consistent feature of Goldenhar syndrome. Similar 
findings were reported by Tasse et al (65-75\%) and $100 \%$ by Martelli et al. Engiz et al in their 31 cases also observed that $77 \%$ had hemifacial microsmia. Facial asymmetry can be caused by either mandibular hypoplasia in these patients or hemifacial microsmia. In general, hemifacial microsomia refers to underdevelopment of one side of the face, with resulting asymmetry. Specifically, the skull, ear, upper and lower jaws, and soft tissues, including the nerves that help move the facial muscles, may be affected. It varies from very mild to severe involvement. These patients pose a significant challenge for the plastic and maxillafacial surgeons. Various techniques including rib grafts have been used to improve their appearance. In our patients $54.5 \%$ had some degree or form of facial asymmetry due to mandibular hypoplasia, whereas, 3 of the patients also had hemifacial microsomia.

Only $34.5 \%$ of patients in our case series had spinal defects. This figure is significantly less than as shown by Martelli et al (100\%) and Engiz et al (70\%). Spinal defects generally include scoliosis and/or kyphosis, hemivertebrae or cervical fusion ${ }^{6}$.

Polydactyly refers to either an extra finger or an extra toe. It has been documented as a very rare association of Goldenhar syndrome ${ }^{11}$. Isolated cases have been reported in literature. Surprisingly, 4 of our patients $(36.4 \%)$ had polydactyly suggesting that this may not be as rare as previously believed. Two patients had an extra digit in one of their feet whereas 2 had an extra little finger in one hand.

$7-25 \%$ patients have been described as having a cleft lip or palate. Martelli et al, however, have reported a higher percentage (35\%) of patients with a cleft lip or palate. Only 1 (9\%) of our patients had this problem.

None of our patients had cardiac or gastrointestinal effects. Other studies have shown upto $50 \%$ presence of cardiac anomalies. Engiz et al and Touliatou et al also reported cardiac defects in $39 \%$ and $18 \%$, respectively, but no cardiac defect was found in patients described by Martelli et al. These defects include ventricular septal defects, Fallot's disease and others. The importance of proper cardiological evaluation cannot, therefore, be overemphasized.

Gastrointestinal defects have been reported occasionally in Goldenhar syndrome and may cause feeding difficulties. Only one patient was reported by Martelli et al in their case series to have gastrointestinal abnormality, whereas $12 \%$ had the same in the study done by Touliatou et al.

Various genitourinary defects have been associated with oculo-auriculo-vertebral disease. These include usually unilateral renal agenesis and renal hypoplasia and cryptorchidism. Only one of our patients had unilateral renal hypoplasia. No genitourinary defect was seen in any of the other patients. 33\% of patients seen by Martelli et al and $23 \%$ each in those seen by Engiz eta al and Touliatou et al had some form of genitourinary abnormality.

One of our patients had VII nerve palsy. Facial nerve palsy is one of the common neurological manifestations of this disorder (12\% reported by Touliatou et al). Facial nerve involvement is thought to be due to atrophic changes in association with hemifacial microsomia or mandibular hypoplasia. In the same study conducted by Touliatou et al $23 \%$ patients also had mental retardation whereas only one (9\%) of our patients had mental retardation. Engiz et al demonstrated central nervous system malformations in $47 \%$ of patients. These malformation or abnormalities generally, include Facial nerve palsy, cerebral anomalies, agenesis of corpus callosum, hypoplasia of encephalic pons, hydrocephalus, microcephaly, encephalocele, etc. Many of these may result in various forms of mental insufficiency or retardation. 


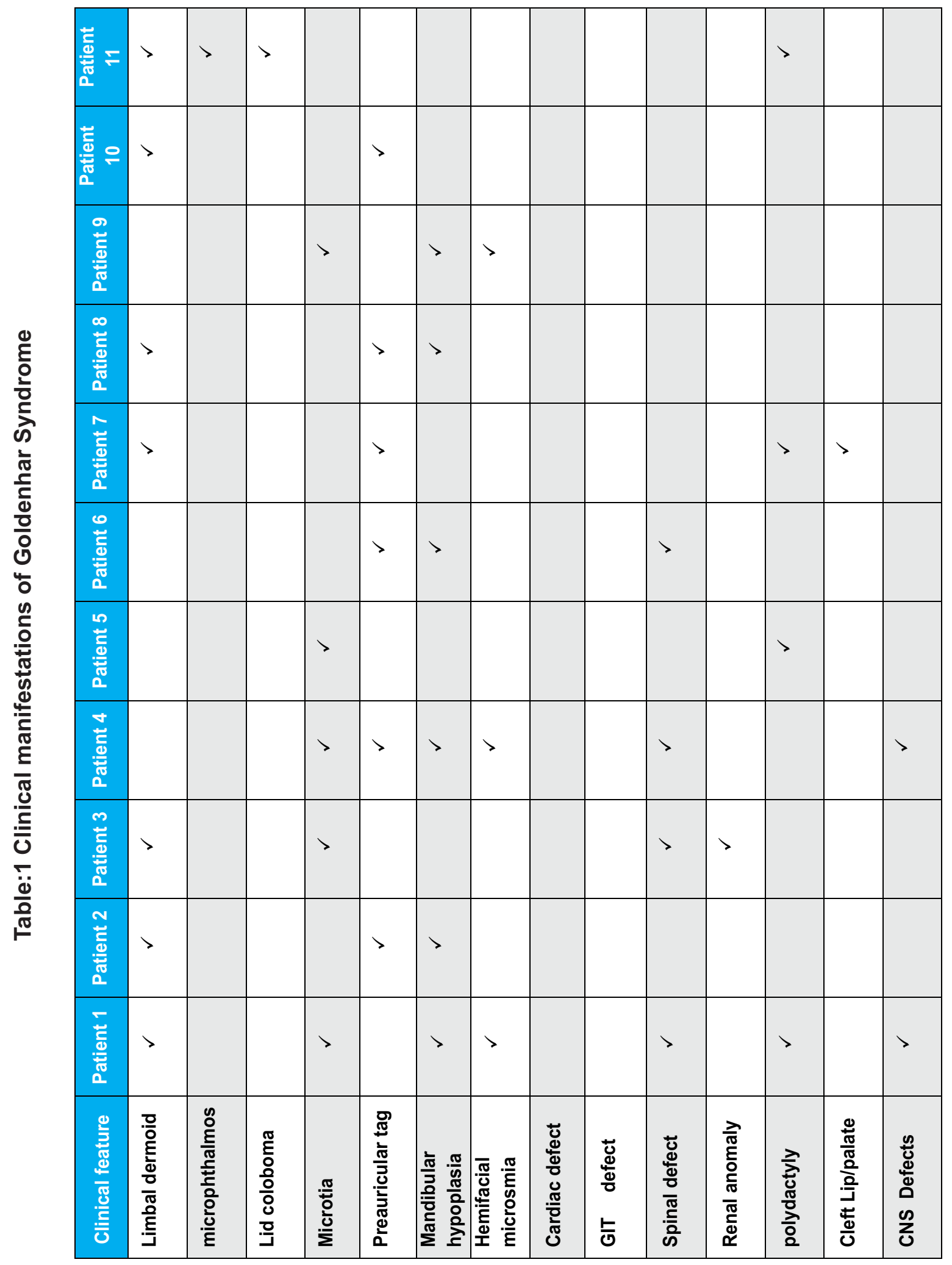




\section{CONCLUSION}

In our study we were able to document the variable manifestations of Goldenhar and their frequency. We also established a definite multisystem spectrum of the disease. Our study correlates with other case series found in contemporary literature. This knowledge base will be helpful in planning treatment and assistance strategies which are complex both for the great variability among patients and for the multiorgan involvement, requiring a multidisciplinary approach.

\section{Copyright (c) 15-06-2013}

\section{REFERENCES}

1. Anderson PJ, David DJ. Spinal anomalies in Goldenhar Syndrome. Cleft Palate Craniofac J. 2005; 42:477-80.

2. Rollnick BR, Kaye Cl, Nagatoshi K, Hauck W, Martin AO. Oculoauriculovertebral dysplasia and variants: phenotypic characteristics of 294 patients. Am J Med Genet. 1987; 26:361-75.

3. Kokavec R. Goldenhar syndrome with various clinical manifestations. Cleft Palate Craniofac J. 2006; 43:628-34.

4. Tasse C, Bohringer S, Fischer S, Ludecke HJ, Albrecht $B$, Horn D, et al. Oculo-auriculo-vertebral spectrum (OAVS): clinical evaluation and severity scoring of 53 patients and proposal for a new classification. Eur $\mathrm{J}$ Med Genet. 2005; 48:397-411.
5. Touliatou V, Fryssira H, Mavrou A, Kanavakis E, KitsiouTzeli S. Clinical manifestations in 17 Greek patients with Goldenhar syndrome. Genet Couns. 2006; 17:359-70.

6. Vendramini S, Richieri-Costa A, Guion-Almeida ML. Oculoauriculovertebral spectrum with radial defects: a new syndrome or an extension of the oculoauriculovertebral spectrum? Report of fourteen Brazilian cases and review of the literature. Eur J Hum Genet. 2007;15:411-21.

7. Hartsfield JK. Review of the etiologic heterogeneity of the oculo-auriculo-vertebral spectrum (Hemifacial Microsomia). Orthod Craniofac Res. 2007; 10:121-8.

8. Engiz 0. Balci S. Unsal M. Ozer S. Oguz K. K. Aktas D. 31 cases with oculoauriculovertebral dysplasia (goldenhar syndrome) : Clinical, neuroradiologic, audiologic and cytogenetic findings. Genetic counseling. 2007: 18(3) : 277-288

9. Martelli H, Miranda RT, Fernandes CM, Bonan PRF. Goldenhar syndrome: clinical features with orofacial emphasis. J Appl Oral Sci. 2010;18(6):646-9

10. Stromland K, Miller $M$, Sjogreen $L$, Johansson $M$, Joelsson BM, Billstedt $E$, et al. Oculo-auriculovertebral spectrum: associated anomalies, functional deficits and possible developmental risk factors. Am J Med Genet A. 2007; 143A:1317-25.

11. Mishra D, Sinha BP, Kumar R. Goldenhar syndrome with unusual association of pre-axial polydactyly. Eur J Ophthalmol. 2009; 19: 1063-4. 
AUTHOR(S):

1. DR. FUAD AHMAD KHAN NIAZI

FCPS, FRCS(Glasg)

Assistant Professor of Ophthalmology

Rawalpindi Medical College, Rawalpindi

2. DR. ABDUL MUNIM KHAN

MBBS, MCPS, FCPS.

Associate Professor of Ophthalmology

Mohtarma Benazir Bhutto Shaheed Medical College, Mirpur

AJK

3. DR. MANAL NIAZI

MBBS, FCPS

Assistant Professor Radiology

Islamabad Medical \& Dental College, Islamabad

\section{Prof. Muhammad Afzal Khan Niazi}

Professor \& Head of Department of Ophthalmology

Wah Medical College, Wah

Correspondence Address:

\section{Dr. Fuad Ahmad Khan Niazi}

FCPS, FRCS(Glasg)

Assistant Professor of Ophthalmology

Rawalpindi Medical College, Rawalpindi

fuadkhan1@yahoo.com

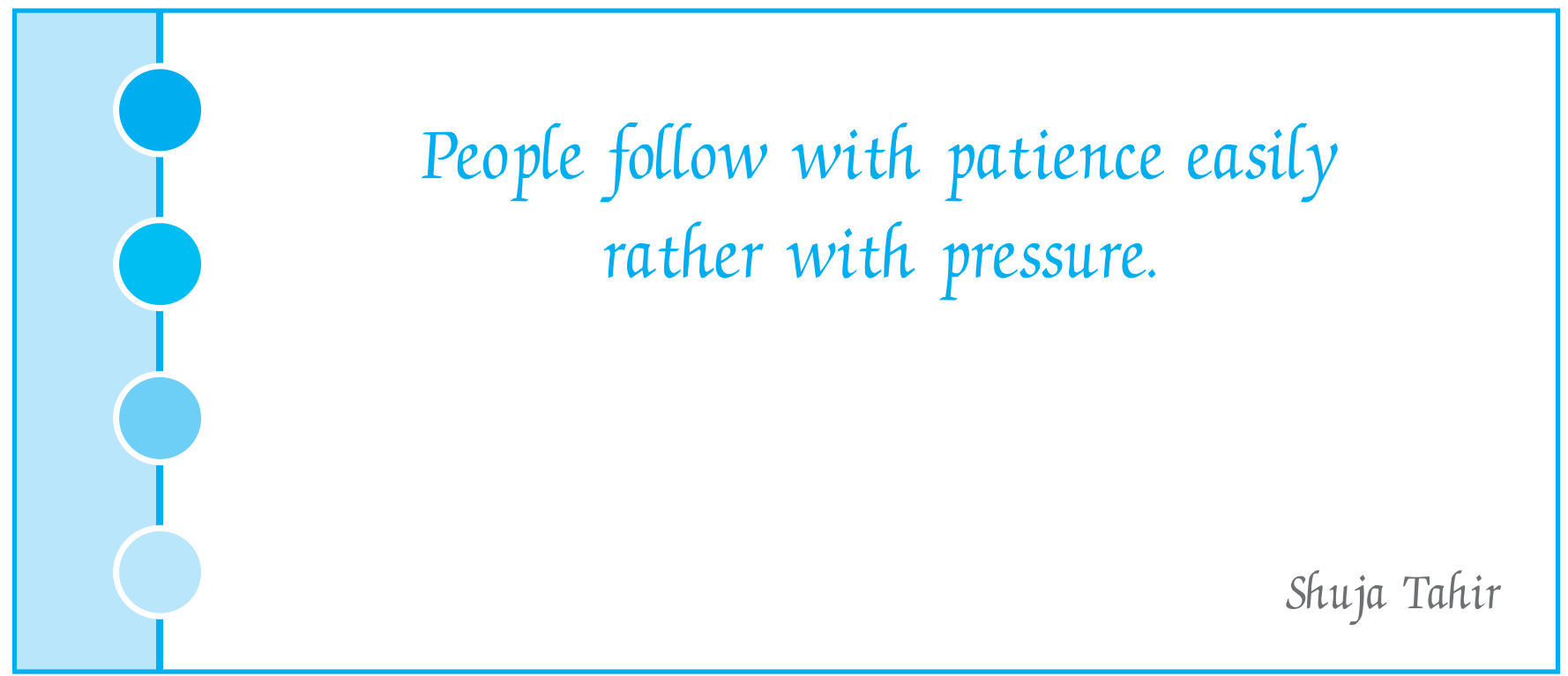

